\title{
Bernstein polynomials method for numerical solutions of integro-differential form of the singular Emden-Fowler initial value problems
}

\author{
Abdelkrim Bencheikh ${ }^{\mathrm{a}}$, Lakhdar Chiter ${ }^{\mathrm{b}, *}$, Hocine Abbassi $^{\mathrm{a}}$ \\ a Department of Mathematics, University Kasdi-Merbah, Ouargla, 30000, Algeria. \\ ${ }^{b}$ Department of Mathematics, Ferhat-Abbas University, Sétif, 19000, Algeria.
}

\begin{abstract}
In this paper, Bernstein polynomial method applied to the solutions of generalized Emden-Fowler equations as singular initial value problems is presented. Firstly, the singular differential equations are converted to Volterra integro-differential equations and then solved by the Bernstein polynomials method. The properties of Bernstein polynomials via Gauss-Legendre rule are used to reduce the integral equations to a system of algebraic equations which can be solved numerically. Some illustrative examples are discussed to demonstrate the validity and applicability of the present method. (C)2017 all rights reserved.
\end{abstract}

Keywords: Bernstein polynomials, Volterra integro-differential equations, Emden-Fowler equation, Gaussian integrations. 2010 MSC: 05C12, 05C15, 05C76.

\section{Introduction}

There exists sufficiently large number of particular basic second-order singular nonlinear ordinary differential equations in mathematical physics and nonlinear mechanics for which an exact analytic solution in terms of known functions did not exist $[2,5]$.

One of these equations describing this type of differential equations is the Emden-Fowler equation formulated as

$$
y^{\prime \prime}+\frac{k}{x} y^{\prime}+\alpha f(x) g(y)=0, \quad k \succeq 0, \quad 0<x \preceq 1, \quad \alpha \succeq 0,
$$

with initial conditions

$$
y(0)=a, \quad y^{\prime}(0)=0,
$$

where $f$ and $g$ are some given functions of $x$ and $y$, respectively.

In mathematical physics and astrophysics, the above equation can model several problems, for example, for $\alpha=1$, it models the heat equation

$$
y^{\prime \prime}+\frac{k}{x} y^{\prime}+f(x) g(y(x))=h(x), k>0, x>0,
$$

\footnotetext{
*Corresponding author

Email address: 1chiter@univ-setif .dz (Lakhdar Chiter)
}

doi:10.22436/jmcs.017.01.06 
where $y(x)$ represents the temperature. For the steady-state case and when $k=2$, and $h(x)=0$, this equation is called the generalized Emden-Fowler equation

$$
y^{\prime \prime}+\frac{2}{x} y^{\prime}+f(x) g(y(x))=0, x>0,
$$

subject to the conditions

$$
y(0)=a, \quad y^{\prime}(0)=b .
$$

By setting $f(x)=1$, the above equation will be reduced to the Lane-Emden equation which models several phenomena in mathematical physics and astrophysics for different values of $g(y(x))$. It is used in theory of stellar structure, theory of thermionic currents, modeling the thermal behavior of a spherical cloud of gas, modeling isothermal gas sphere, and so on.

$$
\begin{gathered}
y^{\prime \prime}+\frac{2}{x} y^{\prime}+g(y(x))=0, x>0 \\
y(0)=a, y^{\prime}(0)=b .
\end{gathered}
$$

For $g(y(x))=y^{p(x)}, a=1$ and $b=0$, this equation is the standard Lane-Emden equation which is used to model thermal behavior of a spherical cloud of gas, acting under the mutual attraction of its molecules, and subject to the classical laws of thermodynamics.

In mathematical physics and astrophysics, equation (1.1) is a basic equation in the theory of stellar structure. It arises in astrophysics and used for computing the structure of interiors of polytropic stars. This equation describes several phenomena such as nuclear physics, theory of thermionic currents, and the thermal variation of a spherical gas cloud under the mutual attraction of its molecules. The EmdenFowler equation appears also in other contexts such as radiative cooling, self-gravitating gas clouds, mean- eld treatment of a phase transition in critical adsorption, modeling of clusters of galaxies, and in study of chemically reacting systems $[4,5,14,15]$. A substantial amount of work has been done on these types of problems for various structures. The singular behavior that occurs at $x=0$ is the main difficulty of these equations.

The solution of Emden-Fowler equations has several known methods. The physical structure of the solutions with a discussion of the formulation can be found in $[2,3,5,10,14,17]$. A general study has been given by Wazwaz [20,21] to construct both exact and series solutions to Emden-Fowler equations through Adomian decomposition method. Also, Yousefi presented this study by Legendre scaling function [22, 23]. To the best of our knowledge, the use of Bernstein polynomials to solve Emden-Fowler equations as singular initial value problems have not been considered in the literature, however, the Lane-Emden equation have been considered in $[9,12,18,19]$ as initial value problems. It is noticed here that Bernstein polynomials have been used in some few restricted problems as singular differential equations, see [1, 6$8,11,13,23]$. In this article, we are concerned with the application of Bernstein polynomials (BPs) to the numerical solution of (1.1). The method consists of convert of Emden-fowler equation to an integrodifferential equation and expanding the solution by BPs with unknown coefficients. The BPs method converts the Volterra integro-differential equation to a system of algebraic equations which can be solved by any of the usual numerical methods. The properties of BPs together with the Gaussian integration formula [4] are then utilized to evaluate the unknown coefficients and an approximate solution to equation (1.1).

The paper is organized as follows. In Section 2, our main work is to establish Volterra integrodifferential equation equivalent to the singular Emden-Fowler initial value problems. In Section 3, we present properties of BPs and approximation of function. In Section 4, we use BPs method integrodifferential form of the singular Emden-Fowler equation. Section 5 illustrates some numerical examples to show the accuracy of this method. Finally, Section 6 concludes the paper. 


\section{Volterra integro-differential form of the singular Emden-Fowler type differential equation}

Consider the Emden-fowler equations given in equation (1.1).

$$
y^{\prime \prime}+\frac{k}{x} y^{\prime}+\alpha h(x) g(y)=0, \quad y(0)=a, \quad y^{\prime}(0)=0, \quad k>1, \quad \alpha>0 .
$$

To convert (2.1) to an integral form, we first set

$$
y(x)=a-\frac{\alpha}{k-1} \int_{0}^{x} t\left(1-\frac{t^{k-1}}{x^{k-1}}\right) h(t) g(y(t)) d t .
$$

Differentiating (2.2) twice, and using the Leibniz rule, we find the integro-differential equation form of the Emden-fowler equation

$$
\begin{aligned}
& y^{\prime}(x)=-\alpha \int_{0}^{x}\left(\frac{t^{k}}{x^{k}}\right) h(t) g(y(t)) d t \\
& y^{\prime \prime}(x)=\alpha h(x) g(y(x))-\alpha \int_{0}^{x} k\left(\frac{t^{k}}{x^{k+1}}\right) f(t) g(y(t)) d t
\end{aligned}
$$

however, for $k=1$ the integral form is

$$
y(x)=a-\alpha \int_{0}^{x} t \ln \left(\frac{t}{x}\right) h(t) g(y(t)) d t,
$$

which can be obtained by setting $k \rightarrow 1$ in equation (2.2). Based on the last results, we set the Volterra integral forms for the Emden-fowler equations as

$$
y(x)=\left\{\begin{array}{l}
a-\alpha \int_{0}^{x} t \ln \left(\frac{t}{x}\right) h(t) g(y(t)) d t \text { for } k=1 \\
a-\frac{\alpha}{k-1} \int_{0}^{x} t\left(1-\frac{t^{k-1}}{x^{k-1}}\right) h f(t) g(y(t)) d t \text { for } k>1
\end{array}\right.
$$

\section{Properties of Bernstein polynomials}

The goal of this section is to recall notations and definitions of the BPs that are used in $[1,6,7,11,13$, $16,23]$.

\subsection{Definitions and properties}

As we mentioned, $m$-degree B-polynomials are a set of polynomials defined on $[0,1]$ by

$$
B_{i, n}(x)=\left(\begin{array}{c}
n \\
i
\end{array}\right) x^{i}(1-x)^{n-i}, \quad i=0,1,2, \ldots, n,
$$

where $\left(\begin{array}{c}n \\ i\end{array}\right)$ means

$$
\frac{n !}{i !(n-i) !} \text {. }
$$

The set of Bernstein polynomials is may be written as a $n+1$-vector $\phi(x)$

$$
\phi(x)=\left[B_{0, n}(x), B_{1, n}(x), \ldots, B_{n, n}(x)\right]^{\top} .
$$

The Bernstein polynomials on $[0,1]$ have the following properties: 
1. The positivity property: For $i=0,1, \ldots, n$, and $x \in[0,1]$, we have

$$
B_{i, n}(x) \geqslant 0 \text {. }
$$

2. The partition of unity property: The binomial expansion of the left-hand side of the equality $(x+$ $(1-x))^{m}=1$ shows that the sum of all Bernstein basis polynomials of degree $m$ is the constant 1 , i.e.,

$$
\sum_{s=1}^{n} B_{i, n}(x)=1 \text {. }
$$

3. By using the binomial expansion of $(1-x)^{m-i}$, one can show that

$$
\left(\begin{array}{c}
n \\
i
\end{array}\right) x^{i}(1-x)^{n-i}=\sum_{k=1}^{n-i}(-1)^{k}\left(\begin{array}{c}
n \\
i
\end{array}\right)\left(\begin{array}{c}
n-i \\
k
\end{array}\right) x^{i+k} .
$$

4. It has a degree raising property in the sense that any of the lower-degree polynomials (degree $<n$ ) can be expressed as a linear combinations of polynomials of degree $n$. We have

$$
B_{i, n-1}(x)=\left(\frac{n-i}{n}\right) B_{i, n}(x)+\left(\frac{i-i}{n}\right) B_{i-1, n}(x) .
$$

5. $\phi(x)$ can be written in the form

$$
\phi(x)=A_{n} \times T_{n}(x),
$$

where

$$
T_{n}(x)=\left[x^{0}, x^{1}, \ldots, x^{n}\right]^{t}
$$

and the $(i+1)$-th row of matrix $A$ is

$$
A_{n}=\left[\begin{array}{cccc}
(-1)^{0}\left(\begin{array}{c}
n \\
0
\end{array}\right) & (-1)^{1}\left(\begin{array}{c}
n \\
0
\end{array}\right)\left(\begin{array}{c}
n-0 \\
1
\end{array}\right) & \ldots & (-1)^{m-0}\left(\begin{array}{c}
n \\
0
\end{array}\right)\left(\begin{array}{c}
n-0 \\
n-0
\end{array}\right) \\
\vdots & \ddots & \ddots & \vdots \\
0 & (-1)^{0}\left(\begin{array}{c}
n \\
i
\end{array}\right) & \ldots & (-1)^{m-i}\left(\begin{array}{c}
n \\
i
\end{array}\right)\left(\begin{array}{c}
n-i \\
m-i
\end{array}\right) \\
\vdots & \vdots & \ddots & \vdots \\
0 & 0 & \cdots & (-1)^{0}\left(\begin{array}{c}
n \\
n
\end{array}\right)
\end{array}\right]
$$

and $\left|A_{n}\right|=\prod_{i=0}^{n}\left(\begin{array}{c}n \\ i\end{array}\right)$, so $A$ is an invertible matrix.

\subsection{Function approximation}

\subsubsection{Approximation of $\mathrm{f}(\mathrm{x})$}

A function $f(x)$, square integrable in $[0,1]$, may be expressed in terms of the Bernstein basis. In practice, only the first- $(n+1)$-terms Bernstein polynomials are considered. Hence if we write

$$
f(x)=\sum_{s=1}^{n} c_{i} B_{i, n}(x)=C^{\top} \phi(x)=C^{\top} \times A_{n} \times T_{n}(x),
$$

where

$$
C^{\top}=\left[c_{0}, c_{1} \ldots, c_{n}\right], \quad \phi(x)=\left[B_{0, n}(x), B_{1, n}(x), \ldots, B_{n, n}(x)\right]^{\top}=A_{n} \times T_{n}(x),
$$

then

$$
C=Q^{-1}\langle f(x), \phi(x)\rangle,
$$


where $Q$ is an $(n+1) \times(n+1)$ matrix, and is said the dual matrix of $\phi(x)$

$$
\begin{aligned}
Q=\langle\phi(x), \phi(x)\rangle=\int_{0}^{1} \phi(x) \phi^{\top}(x) d x=\int_{0}^{1} A_{n} \times T_{n}(x)\left(A_{n} \times T_{n}(x)\right)^{\top} d x & =A_{n} \int_{0}^{1} T_{n}(x) T_{n}(x)^{\top} d x A_{n}^{\top} \\
& =A_{n} H A_{n}^{\top},
\end{aligned}
$$

where $\mathrm{H}$ is a Hilbert matrix

$$
H=\left[\begin{array}{cccc}
1 & \frac{1}{2} & \cdots & \frac{1}{n+1} \\
\frac{1}{2} & \frac{1}{3} & & \frac{1}{n+2} \\
\vdots & \vdots & \ddots & \vdots \\
\frac{1}{n+1} & \frac{1}{n+2} & \cdots & \frac{1}{2 n+1}
\end{array}\right]
$$

The elements of the dual matrix $\mathrm{Q}$ are given explicitly by

$$
Q_{i+1, j+1}=\int_{0}^{1} B_{i, n}(x) B_{j, n}(x) d x=\left(\begin{array}{c}
n \\
i
\end{array}\right)\left(\begin{array}{c}
n \\
j
\end{array}\right) \int_{0}^{1}(1-x)^{2 n-i-j} x^{i+j} d x=\frac{\left(\begin{array}{c}
n \\
i
\end{array}\right)\left(\begin{array}{c}
n \\
j
\end{array}\right)}{(2 n+1)\left(\begin{array}{c}
2 n \\
i+j
\end{array}\right)},
$$

where $i, j=0,1, \ldots, n$.

\subsubsection{Approximate of $\mathrm{g}(\mathrm{y}(\mathrm{x}))$}

We can also approximate the function $g(y(x))$ by the Bernstein polynomials as

$$
\begin{gathered}
g(y(x)) g\left(C^{\top} \phi(x)\right), \\
G^{\top} \phi(x),
\end{gathered}
$$

where the unknown is $G^{\top}=\left[d_{0}, d_{1}, \ldots, d_{n}\right]$. Similarly, we have $G=Q^{-1} \int_{0}^{1} g\left(C^{\top} \phi(x)\right) \phi^{\top}(x) d x$.

\section{Bernstein polynomials method for integro-differential form of the singular Emden-Fowler equation}

Consider the Volterra integro-differential equation given in (2.3) which is the form of Emden-Fowler equation defined in (2.1). To apply the BPs, we first approximate the unknown function $y(x)$ as

$$
y(x)=C^{\top} \phi(x)
$$

where $C$ is defined similar to (3.1).

Integrating (2.3) and using the initial condition $y(0)=1$, we have

$$
y(x)=a-\alpha \int_{0}^{x}\left[\int_{0}^{z}\left(\frac{t^{k}}{z^{k}}\right) h(t) g(y(t)) d t\right] d z, \quad k \succeq 1 .
$$

Using (4.1) and (4.2), we get

$$
\begin{aligned}
C^{\top} A_{n} T_{n}\left(x_{i}\right) & =a-\alpha \int_{0}^{x}\left[\int_{0}^{z}\left(\frac{t^{k}}{z^{k}}\right) h(t) g\left(C^{\top} \phi(t)\right) d t\right] d z, \quad k \succeq 1 \\
& =a-\alpha G^{\top} A_{n} \int_{0}^{x} \int_{0}^{z}\left(\frac{t^{k}}{z^{k}}\right) h(t) T_{n}(t) d t d z \\
& =a-\alpha G^{\top} A_{n} \int_{0}^{x} H(z) d z
\end{aligned}
$$


where

$$
H(z)=\int_{0}^{z}\left(\frac{t^{k}}{z^{k}}\right) h(t) T_{n}(t) d t .
$$

We use the collocation points defined by $x_{i}=\frac{i}{2 n-1}, i=1,2, \ldots . n$

$$
C^{\top} A_{n} T_{n}\left(x_{i}\right)=1-\alpha G^{\top} A_{n} \int_{0}^{x_{i}} H(z) d z .
$$

To use the Gaussian integration formula for (4.3), we transform the interval $\left[0, x_{i}\right]$ into the interval $[-1,1]$ by means of the transformation

$$
\tau=\frac{2}{x_{i}} z-1
$$

Equation (4.3) can be written as

$$
C^{\top} A_{n} T_{n}\left(x_{i}\right)=a-\frac{\alpha x_{i}}{2} G^{\top} A_{n} \int_{-1}^{1} H\left(\frac{x_{i}}{2}(\tau+1)\right) d \tau .
$$

Using the Gaussian integration formula, we obtain

$$
C^{\top} A_{n} T_{n}\left(x_{i}\right)=a-\frac{\alpha x_{i}}{2} G^{\top} A_{n} \sum_{j=1}^{s} \omega_{j} H\left(\frac{x_{i}}{2}\left(\tau_{j}+1\right)\right),
$$

where $\tau_{j}$ are the $s$ zeros of the Legendre polynomials $P_{s+1}$ and $\omega_{j}$ are the corresponding weights. The idea behind the above approximation is the exactness of the Gaussian integration formula for polynomials of degree not exceeding $2 s+1$. Equation (4.4) gives a system of $n+1$ nonlinear algebraic equations with same number of unknowns for coefficient matrix C. Solving this system numerically by Newton's method, we can get the values of unknowns for $C$ and hence we obtain the solution $y(x)=C^{\top} \phi(x)$.

\section{Illustrative examples}

Example 5.1 (Emden-Fowler Equation with $f(x)=x^{p}, g(u)=y^{n}$, and $k=2$ ). Consider the Emden-fowler equation given in [20] by

$$
\begin{aligned}
y^{\prime \prime}(x)+\frac{k}{x} y^{\prime}(x)+x^{p} y^{m}(x) & =0, \quad 0<x \preceq 1, \\
y(0)=1, \quad y^{\prime}(0) & =0 .
\end{aligned}
$$

This equation is equivalent to the following integro-differential equation

$$
y^{\prime}(x)=-\int_{0}^{x}\left(\frac{t^{k}}{x^{k}}\right) t^{p} y^{m}(t) d t, \quad y(0)=1 .
$$

1. For $p=m=0$, and $k=2$, the above equation have an exact solution

$$
y(x)=1-\frac{1}{3 !} x^{2}
$$

by applying this method, and taking $n=2$, we find

$$
y(x)=C^{\top} \phi(x)=C^{\top} A_{2} T_{2}(x)=\left[c_{1}, c_{2}, c_{3}\right]\left[\begin{array}{ccc}
1 & -2 & 1 \\
0 & 2 & -2 \\
0 & 0 & 1
\end{array}\right]\left[\begin{array}{c}
1 \\
x \\
x^{2}
\end{array}\right]
$$


So we get

$$
C^{\top} A_{2} T_{2}\left(x_{I}\right)=1-\frac{x_{i}}{2} G^{\top} A_{2} \sum_{j=1}^{s} \omega_{j} H\left(\frac{x_{i}}{2}\left(\tau_{j}+1\right)\right)
$$

then

$$
\left[\mathrm{c}_{0}, \mathrm{c}_{1}, \mathrm{c}_{2}\right]\left[\begin{array}{ccc}
1 & -2 & 1 \\
0 & 2 & -2 \\
0 & 0 & 1
\end{array}\right]\left[\begin{array}{c}
1 \\
x \\
x^{2}
\end{array}\right]=1-\frac{x_{i}}{2}[1,1,1]\left[\begin{array}{ccc}
1 & -2 & 1 \\
0 & 2 & -2 \\
0 & 0 & 1
\end{array}\right] \sum_{j=1}^{s} \omega_{j} \mathrm{H}\left(\frac{x_{i}}{2}\left(\tau_{j}+1\right)\right) .
$$

By taking $j=2$, we have $\omega_{j}=1, \tau_{j}= \pm \sqrt{\frac{1}{3}}$, and then

$$
c_{0}\left(1-2 x_{i}+x_{i}^{2}\right)+c_{1}\left(2 x_{i}-2 x_{i}^{2}\right)+c_{2} x_{i}^{2}=1-\frac{1}{6} x_{i}^{2} .
$$

Finally, we obtain the system

$$
\Leftrightarrow\left\{\begin{array}{l}
c_{1}-1=0 \\
\frac{4}{9} c_{1}+\frac{4}{9} c_{2}+\frac{1}{9} c_{3}-\frac{53}{54}=0, \\
\frac{1}{9} c_{1}+\frac{4}{9} c_{2}+\frac{4}{9} c_{3}-\frac{25}{27}=0,
\end{array} \Leftrightarrow c_{0}=1, c_{1}=1, c_{2}=\frac{5}{6} .\right.
$$

Hence, the solution is

$$
y(x)=C^{\top} \phi(x)=\left(1,1, \frac{5}{6}\right)\left[\begin{array}{ccc}
1 & -2 & 1 \\
0 & 2 & -2 \\
0 & 0 & 1
\end{array}\right]\left[\begin{array}{c}
1 \\
x \\
x^{2}
\end{array}\right]=1-\frac{1}{3 !} x^{2}
$$

which is the exact solution.

2. For $p=0, m=1$, and $k=2$, equation (5.1) is equivalent to the integro-differential equation

$$
y^{\prime}(x)=-\int_{0}^{x}\left(\frac{t^{2}}{x^{2}}\right) y(t) d t, \quad y(0)=1
$$

which has the exact solution

$$
y(t)=\frac{\sin x}{x}
$$

Now, using the Taylor's expansion, we will have

$$
y(t)=\frac{\sin x}{x}=1-\frac{1}{3 !} x^{2}+\frac{1}{5 !} x^{4}-\frac{1}{7 !} x^{6}+\cdots .
$$

By applying this method, and taking $n=4$, we find the following system

$$
\left\{\begin{array}{l}
c_{0}-1=0, \\
0.54232 c_{0}+0.36059 c_{1}+9.0065 \times 10^{-2} c_{2}+1.0003 \times 10^{-2} c_{3}+4.1670 \times 10^{-4} c_{4}-1=0, \\
0.26790 c_{0}+0.42098 c_{1}+0.25121 c_{2}+6.6841 \times 10^{-2} c_{3}+6.6768 \times 10^{-3} c_{4}-1=0, \\
0.11934 c_{0}+0.33106 c_{1}+0.36507 c_{2}+0.18126 c_{3}+3.3883 \times 10^{-2} c_{4}-1=0, \\
5.0653 \times 10^{-2} c_{0}+0.1992 c_{1}+0.37244 c_{2}+0.32467 c_{3}+0.10745 c_{4}-1=0,
\end{array}\right.
$$

which has the solution

$$
\mathrm{C}=\left[\begin{array}{l}
\mathrm{c}_{0} \\
\mathrm{c}_{1} \\
\mathrm{c}_{2} \\
\mathrm{c}_{3} \\
\mathrm{c}_{4}
\end{array}\right]=\left[\begin{array}{c}
1 \\
1 \\
0.97222 \\
0.91669 \\
0.84154
\end{array}\right]
$$


So, in this case the approximate of $y(x)$ is

$$
y_{4}(t)=1+2.8018 \times 10^{-6} x-0.16671 x^{2}+1.7196 \times 10^{-4} \chi^{3}+0721 \times 10^{-3} \chi^{4} .
$$

Again, by applying this method, and taking $n=6$, we find

$$
\mathrm{C}=\left[\begin{array}{l}
\mathrm{c}_{0} \\
\mathrm{c}_{1} \\
\mathrm{c}_{2} \\
\mathrm{c}_{3} \\
\mathrm{c}_{4} \\
\mathrm{c}_{5} \\
\mathrm{c}_{6}
\end{array}\right]=\left[\begin{array}{c}
1 \\
1 \\
0.98889 \\
0.96667 \\
0.93389 \\
0.89167 \\
0.84147
\end{array}\right]
$$

In this case, the approximate of $y(x)$ is

$$
\begin{aligned}
y_{6}(t)= & 1-2.1171 \times 10^{-9} x-0.16667 x^{2}-5.4837 \times 10^{-7} \chi^{3} \\
& +8.3359 \times 10^{-3} \chi^{4}-6.0401 \times 10^{-6} \chi^{5}-1.9239 \times 10^{-4} \chi^{6} .
\end{aligned}
$$

It is observed that, if $\mathrm{n}$ increases, the approximate solution gets closer to the exact solution.

Table 1: Approximate, exact solutions for Example 5.1, and the absolute error.

\begin{tabular}{|c|cl|c|c|c|}
\hline$x$ & $\begin{array}{c}\text { Present method with } \\
\mathrm{n}=4, \quad \mathrm{n}=6,\end{array}$ & $\begin{array}{c}\text { Exact } \\
\text { solution }\end{array}$ & $\begin{array}{c}\text { Absolute error } \\
\text { with } \mathrm{n}=4\end{array}$ & $\begin{array}{c}\text { Absolute error } \\
\text { with } \mathrm{n}=6\end{array}$ \\
\hline 0.0 & 1.00000 & 1.00000 & 1.00000 & 0 & 0 \\
\hline 0.1 & 0.99833 & 0.99833 & 0.99833 & $3.6836 \times 10^{-8}$ & $2.9575 \times 10^{-12}$ \\
\hline 0.2 & 0.99335 & 0.99335 & 0.99334 & $2.6704 \times 10^{-8}$ & $2.3597 \times 10^{-12}$ \\
\hline 0.3 & 0.98507 & 0.98507 & 0.98506 & $7.8329 \times 10^{-9}$ & $2.9622 \times 10^{-12}$ \\
\hline 0.4 & 0.97355 & 0.97355 & 0.97354 & $2.0436 \times 10^{-8}$ & $6.1969 \times 10^{-12}$ \\
\hline 0.5 & 0.95885 & 0.94107 & 0.95885 & $7.3683 \times 10^{-8}$ & $2.7311 \times 10^{-11}$ \\
\hline 0.6 & 0.94107 & 0.94107 & 0.94107 & $1.6722 \times 10^{-7}$ & $5.3400 \times 10^{-10}$ \\
\hline 0.7 & 0.92031 & 0.92031 & 0.92031 & $2.3365 \times 10^{-6}$ & $8.5175 \times 10^{-9}$ \\
\hline 0.8 & 0.89671 & 0.89670 & 0.89669 & $9.9332 \times 10^{-6}$ & $5.2005 \times 10^{-8}$ \\
\hline 0.9 & 0.87039 & 0.87036 & 0.87036 & $2.9239 \times 10^{-5}$ & $2.1082 \times 10^{-7}$ \\
\hline 1.0 & 0.84154 & 0.84147 & 0.84147 & $7.0313 \times 10^{-5}$ & $6.6921 \times 10^{-7}$ \\
\hline
\end{tabular}

Example 5.2 (Emden-Fowler equation where $\left.\alpha=-2, h(x)=2 x^{2}+3, g(y(x))=y(x)\right)$. Consider the Emden-Fowler equation given in $[4,18,19]$ by

$$
\begin{gathered}
y^{\prime \prime}(x)+\frac{2}{x} y^{\prime}(x)-2\left(2 x^{2}+3\right) y(x)=0, \quad 0<x \preceq 1, \\
y(0)=1, y^{\prime}(0)=0 .
\end{gathered}
$$

This equation is equivalent to the integro-differential equation

$$
y^{\prime}(x)=2 \int_{0}^{x}\left(\frac{t^{2}}{x^{2}}\right)\left(2 t^{2}+3\right) y(t) d t, \quad y(0)=1
$$


This problem has the exact solution $y(x)=e^{x^{2}}$. The approximated solution for $n=4$, and for $n=6$ are as follows:

$$
\begin{aligned}
& y_{4}(t)=0.75364 x^{4}-0.17129 x^{3}+1.0394 x^{2}-2.8695 \times 10^{-3} x+1 \\
& y_{6}(t)=0.27240 t^{6}-0.10901 t^{5}+0.54731 t^{4}-1.0179 \times 10^{-2} t^{3}+1.001 t^{2}-3.9837 \times 10^{-5} t+1
\end{aligned}
$$

Since the exact solution is $y(x)=e^{x^{2}}$, by the Taylor's expansion, we will have

$$
y(x)=e^{x^{2}}=1+x^{2}+\frac{1}{2 !} x^{4}+\frac{1}{3 !} x^{6}+\frac{1}{4 !} x^{8}+\frac{1}{5 !} x^{10}+\cdots
$$

It can be observed that, as $\mathrm{N}$ increases, the approximate solution gets closer to the exact solution.

Table 2: Approximate and exact solutions for Example 5.2

\begin{tabular}{|c|cc|c|c|c|}
\hline$x$ & $\begin{array}{r}\text { Present method with } \\
\mathrm{n}=4, \quad \mathrm{n}=6,\end{array}$ & $\begin{array}{c}\text { Exact } \\
\text { solution }\end{array}$ & $\begin{array}{c}\text { Absolute error } \\
\text { with } \mathrm{n}=4\end{array}$ & $\begin{array}{c}\text { Absolute error } \\
\text { with } \mathrm{n}=6\end{array}$ \\
\hline 0.0 & 1.0000 & 1.0000 & 1.0000 & 0 & 0 \\
\hline 0.1 & 1.0100 & 1.0101 & 1.0101 & $3.9033 \times 10^{-5}$ & $5.2830 \times 10^{-8}$ \\
\hline 0.2 & 1.0408 & 1.0408 & 1.0408 & $2.6883 \times 10^{-5}$ & $4.9297 \times 10^{-8}$ \\
\hline 0.3 & 1.0942 & 1.0942 & 1.0942 & $9.3315 \times 10^{-6}$ & $5.463 \times 10^{-8}$ \\
\hline 0.4 & 1.1735 & 1.1735 & 1.1735 & $2.3722 \times 10^{-5}$ & $1.2887 \times 10^{-7}$ \\
\hline 0.5 & 1.2841 & 1.2840 & 1.2840 & $8.1708 \times 10^{-5}$ & $5.6006 \times 10^{-7}$ \\
\hline 0.6 & 1.4331 & 1.4333 & 1.4333 & $1.9291 \times 10^{-4}$ & $1.1348 \times 10^{-5}$ \\
\hline 0.7 & 1.6295 & 1.6323 & 1.6323 & $2.8206 \times 10^{-3}$ & $1.8745 \times 10^{-4}$ \\
\hline 0.8 & 1.8839 & 1.8965 & 1.8965 & $1.2567 \times 10^{-2}$ & $1.1894 \times 10^{-3}$ \\
\hline 0.9 & 2.2089 & 2.2479 & 2.2479 & $3.8979 \times 10^{-2}$ & $5.0329 \times 10^{-3}$ \\
\hline 1.0 & 2.6189 & 2.7183 & 2.7183 & $9.9395 \times 10^{-2}$ & $1.6757 \times 10^{-2}$ \\
\hline
\end{tabular}

\section{Conclusion}

In this paper, Volterra integro-differential equations equivalent to the Emden-Fowler equation as singular initial value problems have been established. The newly obtained Volterra integro-differential form of Emden-Fowler type equations facilitates the computational work and overcomes the difficulty of the singular behavior at $x=0$. Using this procedure, the integro-differential forms have been reduced to solve a system of algebraic equations. The illustrative examples have been included to demonstrate the validity and applicability of the present technique. These examples also exhibit the accuracy and efficiency of the present method.

\section{References}

[1] M. I. Bhatti, P. Bracken, Solutions of differential equations in a Bernstein polynomial basis, J. Comput. Appl. Math., 205 (2007), 272-280. 1, 3

[2] S. Chandrasekhar, An introduction to the study of stellar structure, Dover Publications, Inc., New York, (1967). 1, 1

[3] M. S. H. Chowdhury, I. Hashim, Solutions of Emden-Fowler equations by homotopy-perturbation method, Nonlinear Anal. Real World Appl., 10 (2009), 104-115. 1

[4] A. Constantinides, Applied numerical methods with personal computers, McGraw-Hill, New York, (1987). 1, 5.2

[5] H. T. Davis, Introduction to nonlinear differential and integral equations, Dover Publications, Inc., New York, (1962). 1,1

[6] E. H. Doha, A. H. Bhrawy, M. A. Saker, Integrals of Bernstein polynomials: an application for the solution of high even-order differential equations, Appl. Math. Lett., 24 (2011), 559-565. 1, 3 
[7] E. H. Doha, A. H. Bhrawy, M. A. Saker, On the derivatives of Bernstein polynomials: an application for the solution of high even-order differential equations, Bound. Value Probl., 2011 (2011), 16 pages. 3

[8] S. Karimi Vanani, A. Aminataei, On the numerical solution of differential equations of Lane-Emden type, Comput. Math. Appl., 59 (2010), 2815-2820. 1

[9] A. Kazemi Nasab, A. Kılıçman, Z. P. Atabakan, W. J. Leong, A numerical approach for solving singular nonlinear LaneEmden type equations arising in astrophysics, New Astron., 34 (2015), 178-186. 1

[10] T. Luo, Z.-P. Xin, H.-H. Zeng, On nonlinear asymptotic stability of the Lane-Emden solutions for the viscous gaseous star problem, Adv. Math., 291 (2016), 90-182. 1

[11] K. Maleknejad, B. Basirat, E. Hashemizadeh, A Bernstein operational matrix approach for solving a system of high order linear Volterra-Fredholm integro-differential equations, Math. Comput. Modelling, 55 (2012), 1363-1372. 1, 3

[12] S. Mall, S. Chakraverty, Chebyshev neural network based model for solving Lane-Emden type equations, Appl. Math. Comput., 247 (2014), 100-114. 1

[13] B. N. Mandal, S. Bhattacharya, Numerical solution of some classes of integral equations using Bernstein polynomials, Appl. Math. Comput., 190 (2007), 1707-1716. 1, 3

[14] A. D. Polyanin, V. F. Zaitsev, Handbook of exact solutions for ordinary differential equations, CRC Press, Boca Raton, FL, (1995). 1

[15] O. W. Richardson, The emission of electricity from hot bodies, Longmans, Green and Company, (1921). 1

[16] T. J. Rivlin, An introduction to the approximation of functions, Blaisdell Publishing Co. Ginn and Co., Waltham, Mass.-Toronto, Ont.-London, (1969). 3

[17] X.-F. Shang, P. Wu, X.-P. Shao, An efficient method for solving Emden-Fowler equations, J. Franklin Inst., 346 (2009), 889-897. 1

[18] Z. Šmarda, Y. Khan, An efficient computational approach to solving singular initial value problems for Lane-Emden type equations, J. Comput. Appl. Math., 290 (2015), 65-73. 1, 5.2

[19] A. M. Wazwaz, A new method for solving singular initial value problems in the second-order ordinary differential equations, Appl. Math. Comput., 128 (2002), 45-57. 1, 5.2

[20] A. M. Wazwaz, Adomian decomposition method for a reliable treatment of the Emden-Fowler equation, Appl. Math. Comput., 161 (2005), 543-560. 1, 5.1

[21] A. M. Wazwaz, Analytical solution for the time-dependent Emden-Fowler type of equations by Adomian decomposition method, Appl. Math. Comput., 166 (2005), 638-651. 1

[22] S. A. Yousefi, Legendre scaling function for solving generalized Emden-Fowler equations, Int. J. Inf. Syst. Sci., 3 (2007), 243-250. 1

[23] S. A. Yousefi, M. Behroozifar, Operational matrices of Bernstein polynomials and their applications, Internat. J. Systems Sci., 41 (2010), 709-716. 1, 3 